\title{
Propensity-matched analysis of two port approach versus three port approach for totally thoracoscopic mitral valve replacement
}

\author{
Jian Liu ${ }^{1 \#}$, Peijian Wei ${ }^{1,2 \#}$, Jiexu Ma ${ }^{1,2 \#}$, Liangzheng Fang ${ }^{1}$, Zhao Chen $^{1}$, Zhongming Cao ${ }^{1}$, Fangzhou Liu ${ }^{1}$, \\ Yanjun Liu ${ }^{1}$, Tong Tan ${ }^{1,2}$, Hongxiang Wu ${ }^{1}$, Huanlei Huang ${ }^{1}$, Jimei Chen ${ }^{1}$, Jian Zhuang ${ }^{1}$, Bin Xie ${ }^{1}$, \\ Huiming Guo ${ }^{1}$
}

${ }^{1}$ Department of Cardiovascular Surgery, Guangdong Provincial Key Laboratory of South China Structural Heart Disease, Guangdong Cardiovascular Institute, Guangdong Provincial People's Hospital (Guangdong Academy of Medical Sciences), Guangzhou, China; ${ }^{2}$ Shantou University Medical College, Shantou, China

Contributions: (I) Conception and design: J Liu, P Wei, J Ma; (II) Administrative support: H Guo; (III) Provision of study materials or patients: H Huang, J Chen, J Zhuang, B Xie, H Guo; (IV) Collection and assembly of data: J Liu, P Wei, J Ma, L Fang, Z Cao, Z Chen, F Liu, Y Liu, T Tan, H Wu; (V) Data analysis and interpretation: J Liu, P Wei, J Ma; (VI) Manuscript writing: All authors; (VII) Final approval of manuscript: All authors.

\#These authors contributed equally to this work.

Correspondence to: Hui-Ming Guo. Department of Cardiovascular Surgery, Guangdong Provincial Key Laboratory of South China Structural Heart Disease, Guangdong Cardiovascular Institute, Guangdong Provincial People's Hospital (Guangdong Academy of Medical Sciences), Guangzhou 510080, China. Email: guohuiming@vip.tom.com.

Background: To objectively evaluate the feasibility, safety, effectiveness and short-term outcome of totally thoracoscopic mitral valve replacement via two port approach, we conducted a retrospective study comparing two port approach with three port approach for mitral valve replacement.

Methods: Data for all thoracoscopic mitral valve replacement were analyzed from Guangdong Cardiovascular Institute between January 1, 2016 and December 31, 2017. To account for selection bias between two port approach and three approach, one-to-one propensity score caliper matching without replacement was performed. The clinical data of the two groups were collected, including preoperative cardiac function, operative data, postoperative complications, and short-term outcome.

Results: A total of 330 patients who underwent totally thoracoscopic mitral replacement via two port or three port from January 1, 2016 to December 31, 2017 were enrolled (two-port group: n=103; threeport group: $n=227$ ). Propensity score matching resulted in 71 matched pairs with improved balance post matching in baseline covariates. The baseline differences between two groups were eliminated ( $P>0.05$ for all baseline variables). The cardiopulmonary bypass time $(\mathrm{min})(154.27 \pm 57.02$ vs. $142.68 \pm 51.33 \mathrm{P}=0.183)$ and the aortic cross-clamp time $(\mathrm{min})(106.99 \pm 106.98$ vs. $90.16 \pm 31.63 \mathrm{P}=0.206)$ in the two-port group were not significantly different from those in the three-port group. No significant difference was observed between the two groups in mechanical ventilation time, duration of intensive care unit stay, or amount of postoperative chest drainage. No perioperative death or re-exploration for bleeding was found in either group. As for other postoperative complications, two groups had the similar rate of lung infection lung infection $(1.41 \%$ vs. $1.33 \%$ $\mathrm{P}=1.000)$ or acute renal failure (1.41\% vs. $1.41 \% \mathrm{P}=1.000)$.

Conclusions: No significant differences in cardiopulmonary bypass time, aortic cross-clamp time, overall operative time, perioperative mortality, or complications were observed between two-port and three-port totally thoracoscopic mitral valve replacement. Two-port totally thoracoscopic mitral valve replacement is a safe, effective, and feasible procedure for mitral valve replacement.

Keywords: Totally thoracoscopic mitral valve replacement; two-port; three-port

Submitted Aug 06, 2020. Accepted for publication Oct 06, 2020.

doi: $10.21037 /$ jtd-20-2901

View this article at: http://dx.doi.org/10.21037/jtd-20-2901 


\section{Introduction}

As a standard procedure for cardiac surgery, median sternotomy provides excellent exposure and allows easy access to the heart and great vessels permitting the full range of mitral intervention to be performed safely and effectively. However, median sternotomy has its drawbacks including extensive surgical trauma, cosmetic problems like long middle scar, more blood loss and blood transfusion, unbearable postoperative pain (1). The trend of cardiac surgery is developing toward minimally invasive cardiac surgery that can achieve the effect of traditional median sternotomy. Attention has first been focused on the choice of surgical approach. Since the emergence of small skin incision valve surgery in the 1950s (2), various surgical methods, such as small upper/lower sternal incision and left/right parasternal incision approach, right anterior mini-thoracotomy approach, have appeared successively (3-5). However, cannulation of the great vessel for cardiopulmonary bypass (CPB) stop us from promoting more minimally invasive cardiac procedure any further. In 1996 in Germany, Fromes et al. proposed a method to establish peripheral cardiopulmonary bypass through cannulation of the internal jugular vein, femoral artery, and femoral vein, to avoid splitting the sternum while achieving the goal of draining blood (6). Professor Carpentier et al. successfully performed the first videoassisted minimally invasive mitral valve surgery through a small incision in the right chest wall in the same year (7). Since that, minimally invasive cardiac surgery has had substantial progress and innovation. The past two decades have seen the development and deployment of videoassisted thoracoscopic cardiac surgery. A variety of MICS procedures have been reported, including hemi-sternotomy, mini-thoracotomy, video-assisted approach, completely thoracoscopic approach and robotic procedure. Currently, minimally invasive thoracoscopic cardiac surgery has become in popularity for its prominent manifestations of reducing surgical trauma, less bleeding and great cosmetic results. Most centers apply minimally invasive videoassisted and three-port method thoracoscopic mitral valve replacement via right anterior lateral small incisions, while a few centers have started to use robot-assisted mitral valve replacement (8). And advancements in imaging and surgical instruments have allowed surgeons to perform more minimally invasive cardiac surgery while the main trend of cardiac surgery is to minimize the incision and surgical trauma and reduce postoperative morbidity and mortality.
Currently, the main stream approach of minimally invasive cardiac surgery still is three-port totally thoracoscopic cardiac surgery.

Minimally invasive thoracoscopic cardiac surgery via three port method can remain the integrity of sternum by avoiding splitting it and reduces surgical trauma and the perioperative blood transfusion rate. Potential advantages include a cosmetically appealing scar, decreased post-operative pain and bleeding, shorter ventilation time and hospital stay, and early return to active life. However, some early studies found that the incidence of postoperative stroke and aortic dissection was significantly higher in these procedures than in conventional MS. Therefore, there are researchers expressing their concerns about the safety of minimally invasive approach (9). Recently, a single-center case report and metaanalysis including a large number of patients published by Sündermann et al. showed that in terms of perioperative mortality and major complications such as stroke and aortic dissection, three port method totally thoracoscopic mitral valve replacement achieved similar clinical prognosis as traditional median sternotomy (10).

Since our center started to perform thoracoscopic cardiac surgery in 2009, the operation volume has been increasing at a rate of $30-50 \%$ per year. We are now capable of performing most cardiac procedures via threeport thoracoscopy under cardiopulmonary bypass, for instance, atrial septal defect repair, ventricular septal defect repair, partial atrioventricular septal defect repair, valvular replacement or repair, surgical resection of atrial myxoma, and some off-pump cardiac surgery. To further reduce the physical and mental trauma of cardiac surgery, our center has pioneered the application of two-port method totally thoracoscopic cardiac surgery and achieved good results due to its advantages of less trauma and better cosmetic effect. There is one fewer auxiliary port in the two-port method than in the three-port method, and the methods of intraoperative exposure and operation in the two-port procedure are modified accordingly. As a novel surgical method, it has certain differences from the threeport method. Our previous research aimed to compare the surgical outcomes after mitral valve replacement via either two-port totally thoracoscopic or traditional median sternotomy surgery between January 2012 and July 2015 in our center and reached a similar conclusion. However, it excluded patients underwent three-port method totally thoracoscopic mitral valve replacement (11). Comparing to three port approach, the feasibility, safety, effectiveness and short-term outcome of totally thoracoscopic mitral valve 
replacement via two port approach hasn't been determined. In other word, we should figure out that which approach is most beneficial to the patients. So, we conducted a retrospective study comparing two port approach with three port approach for mitral valve replacement. It helps us to determine the difference between these two approaches to perform a more minimally invasive cardiac surgery. In this study, Propensity score matching (PSM) was used to eliminate selection bias of the data from patients who underwent mitral valve replacement via three port method versus two port method by balancing covariates, to determine and compare the clinical outcome and short-term mortality of these two approaches. It aimed to evaluate the safety and efficacy of two port method totally thoracoscopic mitral valve replacement and provide a research basis to promote the application of this procedure.

We present the following article in accordance with the STROBE reporting checklist (available at http://dx.doi. org/10.21037/jtd-20-2901).

\section{Methods}

\section{Clinical data}

From January 1, 2016 to December 31, 2017, a total of 330 totally thoracoscopic mitral valve replacements were performed in the Department of Cardiac Surgery of Guangdong Cardiovascular Institute (103 cases in the twoport group and 227 cases in the three-port group). Since the feasibility, safety, effectiveness and short-term outcome of totally thoracoscopic mitral valve replacement via two port approach hasn't been determined, comparing to three-port approach. All patients were randomly divided into two-port group or three-port group due the differences of primary surgeon and their preferences. After screening, a total of 256 patients met the inclusion criteria (82 in the two-port group, 174 in the three-port group). Institutional review board approval from the Ethics Committee of Guangdong Provincial People's Hospital (No. 20160110) was obtained for this analysis with patient consent. The study was conducted in accordance with the Declaration of Helsinki (as revised in 2013).

Inclusion criteria: Surgery subjects were adult patients aged between 18 and 70 years who selected minimally invasive surgery or had cosmetic needs and needed mitral valve replacement due to mitral valve disease (no further subgrouping between patients receiving mechanical valve and biological valve). Tricuspid valvuloplasty or radiofrequency ablation for atrial fibrillation might be performed concurrently. Exclusion criteria: (I) redo mitral valve replacement; (II) emergency surgery; (III) severe thoracic deformities, such as scoliosis, severe pectus excavatum, or funnel chest; (IV) previous history of right thoracic surgery, tuberculosis, pleural tuberculosis, or constrictive pericarditis in which the thoracic and pericardial cavities may have extensive adhesions; (V) aortic or aortic valve disease and need for simultaneous surgery; (VI) coronary artery disease and need for concomitant coronary artery bypass grafting; (VII) preoperative left ventricular ejection fraction (LVEF) $<35 \%$ or low cardiac output syndrome; (VIII) liver and kidney insufficiency or systemic malnutrition; (IX) major vascular diseases or malformations such as in the femoral arteries or veins; and (X) unwillingness to complete follow-up or unwillingness or inability to cooperate in completing the study.

\section{Surgical procedure}

\section{Totally two method thoracoscopic mitral valve replacement}

After achievement of intravenous-inhalation combined anesthesia, the patient was ventilated with a double-lumen endotracheal tube and was in the supine position with the right hemithorax elevated to $15-30^{\circ}$, the right arm raised and secured on an armrest, and abduction of the right leg. External defibrillation electrodes were attached to the left anterior-inferior chest wall and right scapular area in the back. A 28 Fr catheter was inserted into the superior vena cava from the jugular vein by an anesthesiologist for drainage of blood. The probe of the transesophageal echocardiography (TEE) routinely remained in the lumen of the esophagus. After systemic heparinization, the setup of cardiopulmonary bypass was initiated via femoral arterial and venous cannulation through a $2-3 \mathrm{~cm}$ transverse incision in the right groin.

The surgical approach was performed via two ports in the right chest (Figure 1).

(I) main operating port: a $3.0-3.5-\mathrm{cm}$ incision in the fourth intercostal space lateral to the right midclavicular line (for facilitating manipulation of two surgical instruments). (II) Thoracoscopic port: a $1.5-\mathrm{cm}$ incision in the fourth intercostal space at the level of the right midaxillary line (for inserting a camera, a left ventricle vent catheter, and an aortic cross-clamp) (Figure 1). A small incision was made in the third or fourth intercostal space parasternal and lateral to the internal mammary artery for insertion of a minimally 

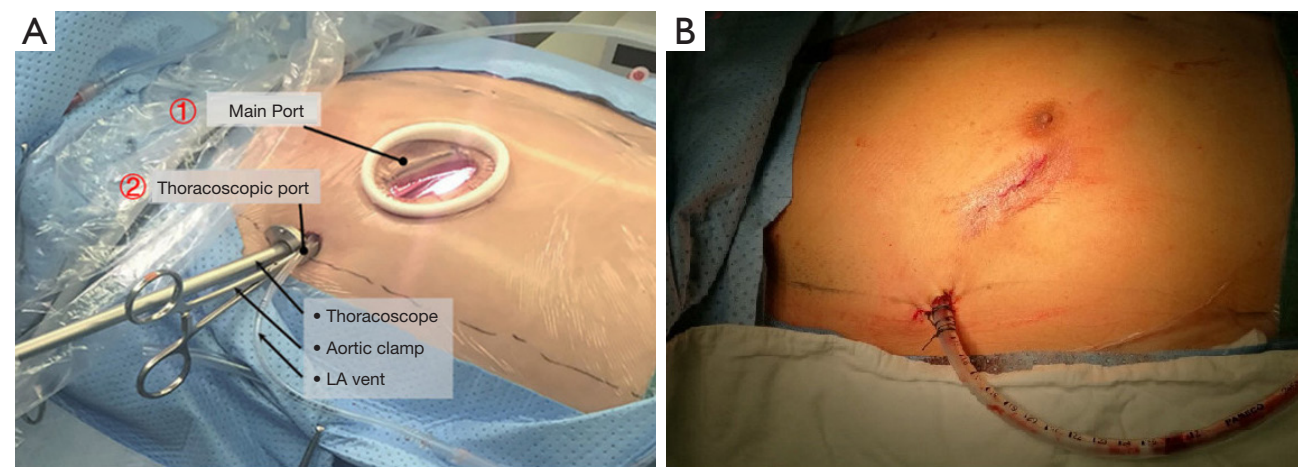

Figure 1 Two-port complete thoracoscopic mitral valve replacement. (A) The port access of two-port complete thoracoscopic mitral valve replacement: (I) main operating port. (II) Thoracoscopic port: thoracoscope, aortic cross-clamp, and left ventricular vent catheter (from top to bottom). (B) The appearance of the incisions after two-port complete thoracoscopic mitral valve replacement.

invasive left atrium automatic retractor.

The right lung was deflated, and carbon dioxide was applied to prevent air embolism as soon as the left atrium was open. The pericardium was opened in front of the right phrenic nerve longitudinally, ranging from the aortic arc to the inferior vena cava, and 3 to 4 sutures were placed to suspend the pericardium. Double pledget purse-string sutures were placed at the root of the ascending aorta, which was prepared for infusing a cardioplegia solution by a perfusion needle to achieve cardiac arrest. Then, aortic cross-clamping were performed through the transverse sinus of the pericardium. Cold crystalloid or cold blood-based cardioplegia solution was used to protect the myocardium via antegrade perfusion. In all patients undergoing mitral valve replacement, the left atrium was opened posterior to the interatrial groove and the mitral valve was exposed via a left atrial retractor Mitral valve replacement was performed routinely under totally endoscopic vision and the prosthetic valve were examined to ensure that they were working well. The left atrium was sutured continuously finally.

Concomitant tricuspid valvuloplasty was performed as necessary after snaring of the superior and inferior vena cava. Through the right atrium, three to four pairs sutures were placed at the free wall to suspend the right atrium for a great vision of the atrial septum, tricuspid valve, and right ventricle, then performed tricuspid valvuloplasty (Kay technique or annuloplasty). After closing the right atrium, the anesthesiologist was asked to reinflate the lung while aspirating the cardioplegia cannula for deairing of the left ventricle. TEE was performed to confirm the position and function of the mitral prosthetic valve after releasing the aortic cross clamp.
The pericardium was closed with interrupted sutures if the outcome was satisfactory and there was no active bleeding at the incisions. Hemostasis of incisions was performed under thoracoscope. After rewarming the patients and inflating the lungs, CPB is discontinued and the patient's heart resumes systemic blood circulation. A thoracic drainage tube was placed through the auxiliary port. Postoperative intercostal nerve block was performed at the level between the $3 \mathrm{rd}$ and 5 th intercostal spaces in the posterior axillary line.

\section{Totally three-port thoracoscopic mitral valve replacement}

Anesthesia preparation, body positioning, and establishment of peripheral cardiopulmonary bypass were same as in the two-port method.

The surgical approach was at the right anterior chest wall: (I) main operating port: a $3.0-3.5-\mathrm{cm}$ incision was made at the anterior axillary line in the fourth intercostal space of the right chest and extended to the midclavicular line (the actual size was chosen to allow insertion of valve prosthesis). The incision was placed just below and lateral to the nipple in male patients, and was placed in the sub-mammary crease in female patients. A medium soft tissue lap-protector was placed over the incision. The aortic perfusion needle and surgical instruments were inserted. (II) Thoracoscopic port: a $1.5-\mathrm{cm}$ incision was made at the 4th intercostal space in the mid-axillary line. A soft tissue lap-protector was placed, and the thoracoscope was inserted. (III) Auxiliary port: a $1.5-\mathrm{cm}$ incision was made at the 5 th intercostal space near the posterior axillary line, and a soft tissue lap-protector was also placed. This incision was for the aortic cross-clamp 

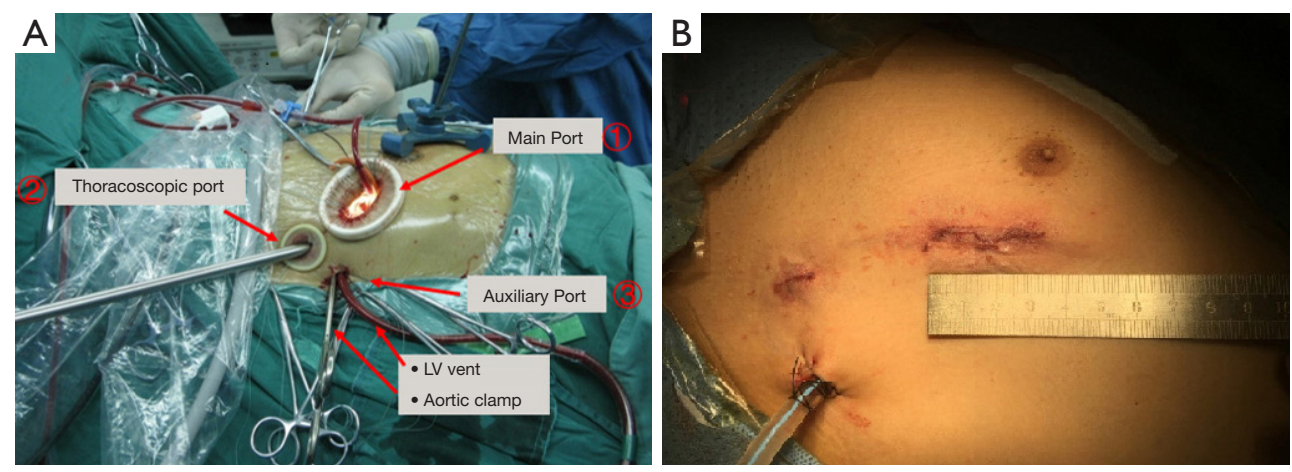

Figure 2 Three-port complete thoracoscopic mitral valve replacement. (A) The port access of Three-port complete thoracoscopic mitral valve replacement. (I) Main operating port. (II) Thoracoscopic port. (III) Auxiliary port: aortic cross-clamp and left ventricle vent catheter (from left to right). (B) The appearance of the incisions after three-port complete thoracoscopic mitral valve replacement.

(Chitwood Clamp), left ventricle vent catheter, and sutures for suspending the pericardium (Figure 2). The rest of the operation was the same as for the two-port method.

Postoperative intercostal nerve block was performed at the level between the $3 \mathrm{rd}$ and 6th intercostal spaces in the posterior axillary line.

\section{Statistical analysis}

All statistical analyses were conducted using SPSS version 21.0 (Version21.0, IBM-SPSS Inc, Armonk, NY, USA). Categorical variables are expressed as the number and percentage of patients, and continuous data are presented as mean \pm standard deviation. Propensity score analysis was utilized to overcome the selection bias attributed to the retrospective nature of this study. Propensity scores were calculated using logistic regression analysis with Age, sex, body mass index (BMI), LVEF, New York Heat Association (NYHA) classification, European System for Cardiac Operative Risk Evaluation II (EuroSCORE II) value, degree of mitral valve lesion, and pulmonary artery pressure as covariates. With the use of the calipermatching method, pairs of patients were matched using a nearest neighborhood (greedy matching) within a caliper width of 0.2 in propensity scores, with a ratio of $1: 1$. This generated 71 pairs. We used the Student's $t$-test to compare continuous variables between two groups. Associations between categorical variables were evaluated by using Chisquared test or Fisher's exact test. $\mathrm{P}$ values $<0.05$ were considered statistically significant for all analyses.

\section{Results}

\section{Preoperative baseline data}

After balancing the baseline data of the two groups, a total of 71 pairs of cases were obtained. A comparative analysis of the baseline data of the two groups showed no significant differences (Table 1).

\section{Intraoperative data}

Both groups of patients successfully underwent mitral valve replacement under general combined anesthesia without any failures. There was no need to extend the incision or convert to median thoracotomy in the two-port group or the three-port group. There was no significant difference between the two groups in cardiopulmonary bypass time, aortic cross-clamp time, or intraoperative blood transfusion rate $(\mathrm{P}>0.05)$ (Table 2).

\section{Postoperative data}

The early postoperative data showed that mechanical ventilation, length of intensive care unit (ICU) stay, the mean volume of chest drainage after surgery, or reexploration for bleeding rate were similar between the 2 matched groups $(\mathrm{P}>0.05)$ (Table 3). No significant difference was detected in the incidence of perioperative mortality or early postoperative complications, such as aortic dissection, stroke, lung infection, arrhythmia, and acute renal failure between the two groups (Table 4). 
Table 1 General clinical data between the two groups after PSM $\left[\left(\bar{x}_{ \pm}\right) / \mathrm{n}(\%)\right]$

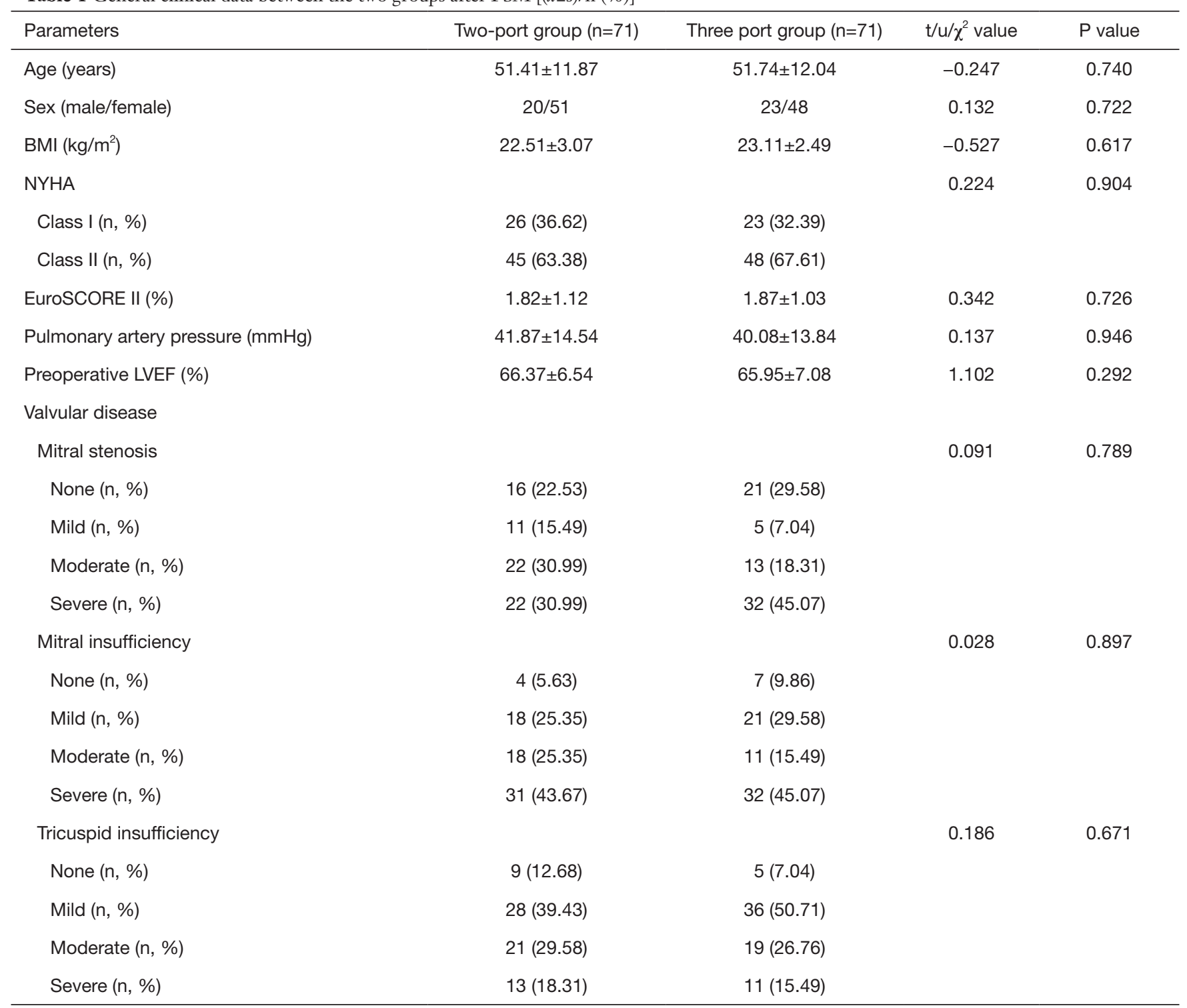

BMI, body mass index; LVEF, left ventricular ejection fraction; NYHA, New York Heart Association Functional Classification; EuroSCORE II, European System for Cardiac Operative Risk Evaluation II.

\section{Discussion}

Minimally invasive thoracoscopic cardiac surgery was performed via a small incision in the right chest wall without the need for median sternotomy. This protects the integrity of the sternum and greatly reduces surgical trauma. This minimally invasive procedure requires a $3-4-\mathrm{cm}$ incision in the chest wall (reduced from a $20-25-\mathrm{cm}$ incision in conventional procedures), with significantly less division of the deep muscles and limited interference with the thymus and other tissues surrounding the heart.
It achieves the goal of minimizing unnecessary trauma. The application of a high-definition thoracoscopy video system and a $10-\mathrm{mm}$ lens provide a precise and clear view of the operative field. This is helpful to find microbleeding during surgery and to perform precise electrocoagulation. It can prevent unnecessary blood loss and the waste of blood resources. It can reduce the risk of blood transfusion-related death and complications, thereby reducing postoperative chest drainage and the chest tube indwelling time. To date, the results reported by many centers in China and abroad 
Table 2 Intraoperative data of patients $\left[\left(\bar{x}_{ \pm s}\right) / \mathrm{n}(\%)\right]$

\begin{tabular}{|c|c|c|c|c|}
\hline Parameter & Two-port group $(\mathrm{n}=71)$ & Three-port group $(n=71)$ & $t$ or $\chi^{2}$ value & $P$ value \\
\hline Aortic cross-clamp time (min) & $108.21 \pm 36.41$ & $98.52 \pm 32.88$ & 1.286 & 0.247 \\
\hline Intraoperative blood transfusion (n, \%) & $11(15.49)$ & $8(11.27)$ & & $0.106^{\#}$ \\
\hline
\end{tabular}

\#, the result from the $\chi^{2}$ test.

Table 3 Early data of postoperative ICU $\left[\left(\bar{x}_{ \pm} \mathrm{s}\right) / \mathrm{n}(\%)\right]$

\begin{tabular}{|c|c|c|c|c|}
\hline Parameters & Two-port group $(\mathrm{n}=71)$ & Three-port group $(n=71)$ & $t$ or $\chi^{2}$ value & $P$ value \\
\hline Length of ICU stay (h) & $70.47 \pm 35.22$ & $53.29 \pm 33.63$ & 1.175 & 0.323 \\
\hline Total amount of chest drainage $(\mathrm{mL})$ & $308.41 \pm 220.15$ & $295.45 \pm 243.64$ & 1.310 & 0.217 \\
\hline Re-exploration of thoracic cavity (n, \%) & $0(0.00)$ & $0(0.00)$ & & $1.000^{\#}$ \\
\hline
\end{tabular}

", the result from the $\chi^{2}$ test.

Table 4 Comparison of major complications in the early postoperative period [ $\mathrm{n}(\%)]$

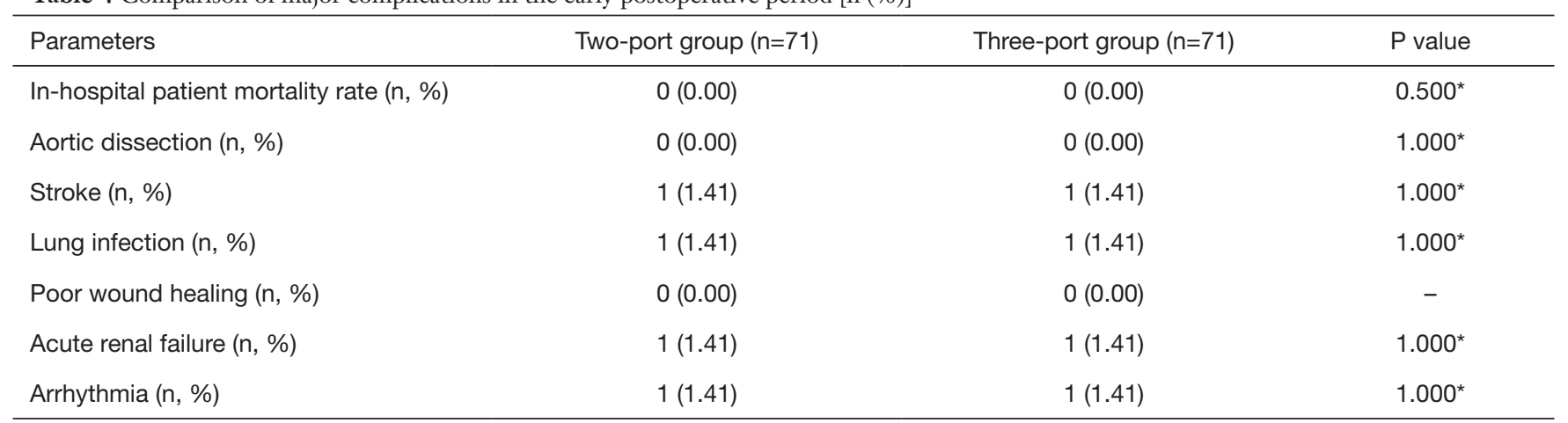

*, calculated by Fisher's exact test.

as well as a meta-analysis indicate that the perioperative mortality and the incidence of serious complications are not significantly different between the mitral valve replacement via three port thoracoscope and via traditional median sternotomy $(10,12,13)$. The three-port method has the advantages of lower hospitalization costs due to the short duration of ICU stay. Our data also support this statement (14). The three-port method totally thoracoscopic mitral valve replacement is a safe and effective procedure (11).

Thoracoscopic surgery is also widely used in thoracic lobectomy. It has evolved from its beginnings as a four-port method to a single-port method today and has gradually become a more minimally invasive procedure (15-17). The study of single-port lobectomy by Gonzalez et al. showed that compared with the multiport method, the single-port method is associated with better postoperative aesthetic outcome and a significant reduction in postoperative pain (18).

In this study, the pioneering application of a two-port method for complete thoracoscopic cardiac surgery was also undertaken to minimize trauma, reducing postoperative pain, and better cosmetic appearance. The one fewer operating port require the method of intraoperative exposure and operation to be modified from the three-port method. Compared with the three-port totally thoracic mitral valve replacement, the greatest challenge of the twoport method is to reduce an auxiliary port that is often used as an approach for left ventricular vent catheter and aortic cross-clamp in minimally invasive cardiac surgery. The modification in the two-port method is to place the aortic cross-clamp through thoracoscopic auxiliary port and the 
left ventricular vent catheter through the main operating port. This modification may cause two problems: (I) the adjustment of the thoracoscope may be limited by the aortic cross-clamp and may change the normal position of the aortic cross-clamp during operation, resulting in torsion and other injuries to the aorta. (II) Due to absence of the second auxiliary port, the left ventricular vent catheter needs to be placed through the main operating port and it leads to the reduction of operating space. Moreover, a conflict of instruments may occur since the instrument and the drainage tube are in a same port. That is, the drainage tube, minimally invasive instruments, and sutures (slings) used for exposure of the operative field can easily interfere with each other, which indirectly increases the operative time.

The following is a summary of our team's experience obtained from continuous exploration: (I) innovative use of a stabilization device for the aortic cross-clamp, such as an endoscopic protective cover: The clamp is placed into the endoscopic port through the stabilization device, which can prevent the clamp from rotating when it reaches the functional position. The thoracoscopic trocar has also been modified. The protective edge of the metal trocar used in this study is cut into a semiarc shape, which can reduce its interaction with the aortic cross-clamp. (II) A puncture needle was used to penetrate the chest wall at the site where the second operating port would be located in the three-port method to introduce the suture for hanging the pericardium out of the thoracic cavity. This method causes less trauma than making a second operating port but can also achieve good visualization of the operative field. The lesser damage to the intercostal muscles can significantly reduce the likelihood of postoperative bleeding because in most cases of reoperation for postoperative bleeding, the bleeding sites are located in the intercostal muscles nourishing blood vessels in the chest wall incision.

Analysis and comparison of postoperative data between the two groups showed that no perioperative death was reported in the 142 patients of this study. There was no significant difference in cardiopulmonary bypass time or aortic cross-clamp time between the two groups. This indicates that the modified two-port method for mitral valve replacement did not increase the operative time. The reason may be that, whether the three-port method or the two-port method was used, the main intracardiac operations were performed through the main operating port, so there was no obvious operational difference between the two groups. In the 142 patients enrolled in this study, no perioperative aortic dissections and stroke were reported. Displacement of the aortic clamp and improper of the perfusion needle may causes the postoperative aortic dissection. In twoport approach, this can be avoided by cautious operations and the trocar can help to limit the movement of the aortic clamp. As for postoperative stroke, the residual intracardiac air may be the leading causes. In two-port approach, the intracardiac air is evacuated through both the atriotomy suture line and aortic vent, which can assure that there's no residual intracardiac air. There was no significant difference in intraoperative blood transfusion, mechanical ventilation, length of ICU stays, rate of re-exploration for bleeding, or amount of postoperative drainage ( $\mathrm{P}>0.05$, Table 3$)$. These data further suggest the two-port method is as safe and effective as the three-port method. No significant difference in the incidence of other early postoperative complications, such as stroke, lung infection, arrhythmia, and acute renal failure were observed between the two groups (Table 4), indicating that the two-port method can be adopted as a safe, effective, and less invasive surgical procedure than the three-port method.

Telephone or outpatient follow-up was performed in all 142 patients ( 71 patients in the two-port group and 71 patients in the three-port group), who were all discharged successfully from the hospital. The follow-up period was between 6 months and 2 years, with an average of $10.51 \pm 5.12$ months. Effective follow-up data were obtained from 139 patients $(2.11 \%$ loss to follow-up). No death was reported during the follow-up. Four patients were diagnosed with paravalvular leakage (2.88\%), including one patent in the two-port group and three patients in the threeport group. No significant difference was found among these results. A retrospective controlled trial conducted by Gumus et al. in 2020 found no significant difference in the incidence of postoperative paravalvular leakage between minimally invasive mitral valve replacement and mitral valve replacement via median thoracotomy (19). The NYHA classification of cardiac function during the follow-up period of the two groups were significantly improved over that before surgery, and there was no significant difference between the two groups. The patients undergoing minimally invasive heart surgery have less pain after surgery and faster recovery. This helps reduce the patient's physical and psychological trauma. Compared with the three-port method, the two-port method reduces the use of soft tissue lap-protector (they are only used on the main operating port). This is conducive to reducing medical costs and improving the utilization rate of medical resources. Patients 
can return to work or study as soon as possible and create more social value and social benefits.

No long-term follow-up data are available in this study, so the long-term efficacy of the two-port method needs to be further verified. Moreover, this study was retrospective, and statistical methods such as PSM can only reduce the bias caused by confounding factors. The efficacy of the two-port method or even minimally invasive thoracoscopic cardiac surgery has yet to be evaluated in large randomized clinical trials.

In summary, two-port thoracoscopic mitral valve replacement can achieve the same effect as the three-port method, and there is no significant difference in the early mortality rate or the incidence of complications such as aortic dissection. Two-port complete thoracoscopic heart surgery is a safe, effective, and feasible procedure.

\section{Acknowledgments}

Funding: None.

\section{Footnote}

Reporting Checklist: The authors have completed the STROBE reporting checklist. Available at http://dx.doi. org/10.21037/jtd-20-2901

Data Sharing Statement: Available at http://dx.doi. org/10.21037/jtd-20-2901

Conflicts of Interest: All authors have completed the ICMJE uniform disclosure form (available at http://dx.doi. org/10.21037/jtd-20-2901). The authors have no conflicts of interest to declare.

Ethical Statement: The authors are accountable for all aspects of the work in ensuring that questions related to the accuracy or integrity of any part of the work are appropriately investigated and resolved. Institutional review board approval from the Ethics Committee of Guangdong Provincial People's Hospital (No. 20160110) was obtained for this analysis with patient consent. The study was conducted in accordance with the Declaration of Helsinki (as revised in 2013).

Open Access Statement: This is an Open Access article distributed in accordance with the Creative Commons Attribution-NonCommercial-NoDerivs 4.0 International
License (CC BY-NC-ND 4.0), which permits the noncommercial replication and distribution of the article with the strict proviso that no changes or edits are made and the original work is properly cited (including links to both the formal publication through the relevant DOI and the license). See: https://creativecommons.org/licenses/by-nc-nd/4.0/.

\section{References}

1. Morgante A, Romeo F. Deep sternal wound infections: a severe complication after cardiac surgery. G Chir 2017;38:33-6.

2. Bailey CP, O'Neill TJ, Glover RP, et al. Surgical repair of mitral insufficiency. Dis Chest 1951;19:125-37.

3. Konertz W, Waldenberger F, Schmutzler M, et al. Minimal access valve surgery through superior partial sternotomy: a preliminary study. J Heart Valve Dis 1996;5:638-40.

4. Nair RU, Sharpe DA. Limited lower sternotomy for minimally invasive mitral valve replacement. Ann Thorac Surg 1998;65:273-4.

5. Zang X, Huang HL, Xie B, et al. A comparative study of three-dimensional high-definition and two-dimensional high-definition video systems in totally endoscopic mitral valve replacement. J Thorac Dis 2019;11:788-94.

6. Fromes Y, Gaillard D, Ponzio O, et al. Reduction of the inflammatory response following coronary bypass grafting with total minimal extracorporeal circulation. Eur J Cardiothorac Surg 2002;22:527-33.

7. Carpentier A, Loulmet D, Carpentier A, et al. Open heart operation under videosurgery and minithoracotomy. First case (mitral valvuloplasty) operated with success. C R Acad Sci III 1996;319:219-23.

8. Ramlawi B, Gammie JS. Mitral Valve Surgery: Current Minimally Invasive and Transcatheter Options. Methodist Debakey Cardiovasc J 2016;12:20-6.

9. Cheng DC, Martin J, Lal A, et al. Minimally invasive versus conventional open mitral valve surgery: a metaanalysis and systematic review. Innovations (Phila) 2011;6:84-103.

10. Sündermann SH, Sromicki J, Rodriguez Cetina Biefer H, et al. Mitral valve surgery: right lateral minithoracotomy or sternotomy? A systematic review and meta-analysis. J Thorac Cardiovasc Surg 2014;148:1989-95.e4.

11. Liu J, Chen B, Zhang YY, et al. Mitral valve replacement via minimally invasive totally thoracoscopic surgery versus traditional median sternotomy: a propensity score matched comparative study. Ann Transl Med 2019;7:341.

12. Modi P, Hassan A, Chitwood WR, Jr. Minimally invasive 
mitral valve surgery: a systematic review and meta-analysis. Eur J Cardiothorac Surg 2008;34:943-52.

13. Cooley DA. Minimally invasive valve surgery versus the conventional approach. Ann Thorac Surg 1998;66:1101-5.

14. Dieberg G, Smart NA, King N. Minimally invasive cardiac surgery: A systematic review and meta-analysis. Int J Cardiol 2016;223:554-60.

15. Kawahara K, Iwasaki A, Shiraishi T, et al. Video-assisted thoracoscopic lobectomy for treating lung cancer. Surg Laparosc Endosc 1997;7:219-22.

16. Gaudet MA, D'Amico TA. Thoracoscopic Lobectomy for Non-small Cell Lung Cancer. Surg Oncol Clin N Am 2016;25:503-13.

Cite this article as: Liu J, Wei P, Ma J, Fang L, Chen Z, Cao Z, Liu F, Liu Y, Tan T, Wu H, Huang H, Chen J, Zhuang J, Xie B, Guo H. Propensity-matched analysis of two port approach versus three port approach for totally thoracoscopic mitral valve replacement. J Thorac Dis 2020;12(10):5986-5995. doi: 10.21037/jtd-20-2901
17. Ikeda N, Saji H, Hagiwara M, et al. Recent advances in video-assisted thoracoscopic surgery for lung cancer. Asian J Endosc Surg 2013;6:9-13.

18. Bédat B, Abdelnour-Berchtold E, Perneger T, et al. Comparison of postoperative complications between segmentectomy and lobectomy by video-assisted thoracic surgery: a multicenter study. J Cardiothorac Surg 2019;14:189.

19. Gumus F, Hasde AI, Bermede O, et al. Multiple Valve Implantation Through a Minimally Invasive Approach: Comparison of Standard Median Sternotomy and Right Anterior Thoracotomy. Heart Lung Circ 2020;29:1418-23. 